\title{
Modelo dinámico de un reactor de ultrasonido continuo en la degradación de anaranjado de metilo
}

Kieffer L.A. • de la Sierra P.M. • Luna J.A. • Lovato M.E. • Claret M.

Para citar este artículo: Kieffer L.A.; De la Sierra P.M.; Luna J.A.; Lovato M.E.; Claret M. (2019). Modelo dinámico de un reactor de ultrasonido continuo en la degradación de anaranjado de metilo. Pág. XX-XX. Santa Fé, Argentina: UNL. DOI 10.14409/ fabicib.v23i0.8019 


\section{MODELO DINÁMICO DE UN REACTOR DE ULTRASONIDO CONTINUO EN LA DEGRADACIÓN DE ANARANJADO DE METILO}

Kieffer L.A. ${ }^{1}$, de la Sierra P.M. ${ }^{2,3}$, Luna J.A. ${ }^{1,3}$, Lovato M.E. ${ }^{3}$ y Claret M. ${ }^{2}$

Ikieffer@santafe-conicet.gov.ar

1 Fundación para la Promoción y Desarrollo Tecnológico del Litoral (VINTEC). Guemes 3450. (3000) Santa Fe

2 Facultad de Ingeniería y Ciencias Hídricas (UNL). Paraje El Pozo. (3000) Santa Fe

3 Instituto de Desarrollo Tecnológico para la Industria Química (UNL - CONICET). Guemes 3450. (3000) Santa Fe

Recibido: 07/03/2019. Aceptado: 11/11/2019

\section{RESUMEN}

Se analiza la degradación de soluciones acuosas del colorante azoico anaranjado de metilo en un reactor sonoquímico de flujo continuo. Las determinaciones de los tiempos de residencia en cada una de las cuatro unidades del reactor muestran que los mismos no difieren de los tiempos espaciales, por lo que el reactor puede considerarse un reactor continuo de mezcla completa sin volúmenes muertos ni cortocircuitos.

Mediante la simulación dinámica se desarrollan modelos de funcionamiento del reactor. Los resultados tanto en el estado transiente como en el estacionario no presentan diferencias estadísticamente significativas con los obtenidos analíticamente.

Palabras clave: ultrasonido, modelos dinámicos, anaranjado de metilo

\section{SUMMARY}

\section{Dynamic model of a continuous ultrasound reactor in the degradation of methyl orange}

In this work, the degradation of aqueous solutions containing the commercial azo dye Methyl Orange in a continuous flow sonochemical reactor was analyzed. The determination of the residence times in each one of the four reactor units showed that they did not differ from the spatial times, consequently the reactor can be considered as a perfectly mixed continuous stirred tank reactor without dead volumes or bypasses. 
Using dynamic simulation techniques, the reactor operation models were developed. The simulation results for both transient and stationary states did not show statistically significant differences with those obtained analytically.

Keywords: ultrasound, dynamic model, methyl orange

\section{INTRODUCCION}

El uso del ultrasonido como método de tratamiento de aguas contaminadas ha mostrado ser una alternativa efectiva, dentro de los procesos de oxidación avanzados, para la degradación de gran cantidad de compuestos refractarios a otros tratamientos $(1-8)$. Pese a la gran cantidad de estudios, la mayoría de ellos son a escala laboratorio en reactores por lotes, mientras que aquellos que utilizan un reactor continuo son escasos.

El anaranjado de metilo (AM) (ácido 4-(((4-Dimetilamino)fenil)azo)benzenosulfónico) es un colorante azoico empleado en la industria textil, muy utilizado en estudios de degradación de contaminantes (9 - 17). Puede causar irritaciones por contacto (piel, ojos), náuseas, diarreas y vómitos si es ingerido (18) y además es considerado un colorante mutagénico (19).

El objetivo de este trabajo es analizar mediante simulación dinámica el funcionamiento de un reactor de ultrasonido continuo utilizando como compuesto modelo anaranjado de metilo, teniendo en cuenta el reactor como un todo y por otro lado analizando sus cuatro unidades por separado, tanto en estado estacionario como durante el transiente.

\section{MATERIALES Y METODOS}

a) Reactor de ultrasonido

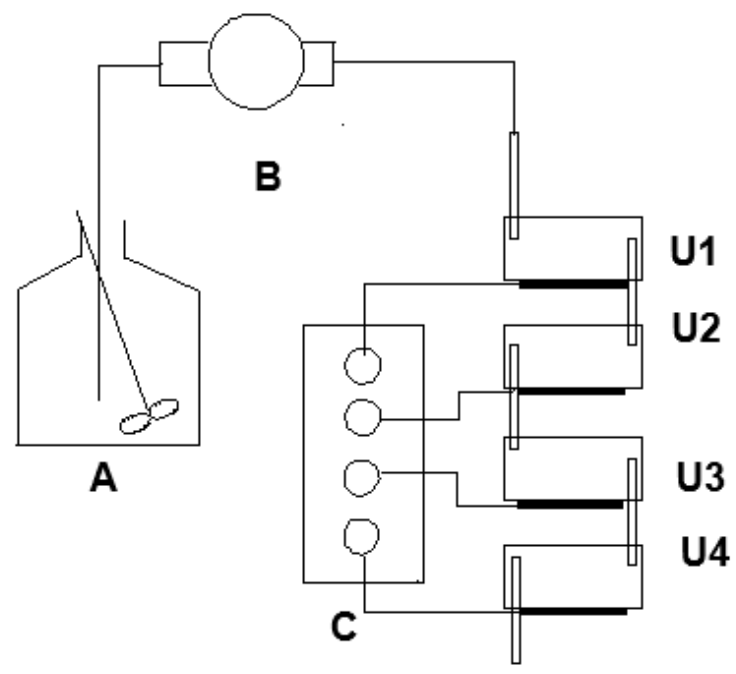

Figura 1. Reactor de ultrasonido continuo: Ui : unidad i; C: control electrónico; B: bomba peristáltica; A: alimentación 
El reactor (Figura 1) está compuesto por cuatro unidades: la primera de 0,530 L (20 kHz y 33,6 W $\pm 0,79$ W), la segunda de $0,526 \mathrm{~L}(40 \mathrm{KHz}$ y $17 \mathrm{~W} \pm 0,75 \mathrm{~W}$ ), la tercera de $0,529 \mathrm{~L}(20 \mathrm{kHz}$ y $27,0 \mathrm{~W} \pm 0,42 \mathrm{~W}$ ) y la ultima de $0,535 \mathrm{~L}$ ( $40 \mathrm{kHz}$ y 49,7 W $\pm 0,23 \mathrm{~W}$ ), donde cada una descarga en la siguiente a través de un rebosadero y conforman un solo cuerpo. La alimentación de las aguas a tratar se efectúa mediante una bomba peristáltica. Cada unidad tiene un sistema de ventilación forzada a fin de mantener temperaturas estables (en los ensayos las mismas variaron entre $24^{\circ} \mathrm{C}$ y $28^{\circ} \mathrm{C}$ ).

\section{b) Reactivos y equipos analíticos}

Se utilizó AM grado analítico Mallinckrodt (PM: 327,34 g/mol, CAS: 547-58-0) y triclorometano pro análisis Cicarelli como sinergizante (PM: 119,38 g/mol, densidad 1,49 g/mL, CAS: 67-66-3) en concentración de $1 \mathrm{ml} / \mathrm{L}$ (20). Para preparar las soluciones se utilizó agua ultrapura (ósmosis inversa - deionizada) de conductividad $<0,05 \mu \mathrm{S} / \mathrm{cm}$. Las concentraciones de AM se midieron por espectrometría visible en un equipo HATCH DR/2010 a 465 nm (21). Todos los ensayos de decoloración se hicieron por triplicado. Los resultados de los ensayos fueron presentados en un trabajo anterior (20). Las determinaciones de $\mathrm{pH}$ se efectuaron con un equipo HATCH Sension PH1.

\section{c) Software utilizado}

Para efectuar los cálculos, ajustes de ecuaciones y simulación dinámica se usó el siguiente software libre:

c1) Open Office 4.1 .5

c2) Curve Expert Professional 2.6

c3) Scilab 6.0.1

\section{d) Planteos teóricos}

\section{d1) Distribución del tiempo de residencia}

En un reactor tanque completamente mezclado ideal, el tiempo promedio que un elemento diferencial de fluido permanece en el reactor se conoce como tiempo espacial o de permanencia, y es igual al cociente entre el volumen del reactor y el caudal. El comportamiento real de un reactor puede desviarse del comportamiento ideal de manera que los tiempos de permanencia sean menores (existencia de canalizaciones o zonas estancadas) o mayores (recirculaciones).

Una manera de determinar el comportamiento del reactor es mediante la determinación de la distribución del tiempo de residencia (DTR). La DTR es una distribución de probabilidades que muestra el tiempo que un elemento de volumen permanece dentro del reactor y es un parámetro para caracterizar la mezcla. 
Experimentalmente, la DTR puede determinarse inyectando un trazador a la entrada del reactor y midiendo su evolución a través del tiempo a la salida del mismo. Los dos métodos más utilizados de inyección son mediante un pulso o un escalón. En éste trabajo se usó el primer método, utilizando como trazador $\mathrm{HCl}$ 1,0 M. Se midió el pH a intervalos de 20 segundos durante 600 segundos, tomando como tiempo inicial el instante en que se inyectó $1 \mathrm{ml}$ de trazador al caudal de entrada $(10 \mathrm{~L} / \mathrm{h})$. Los ensayos se realizaron por triplicado en cada unidad por separado, sin y con los sonotrodos encendidos a fin de verificar también la posible existencia de diferencias por la acción mecánica del ultrasonido sobre el fluido (en nuestro caso se utilizó agua destilada).

La función de distribución del tiempo de residencia $(E(t))$ se define como:

$$
E(t)=\frac{P(t)}{\int_{0}^{\infty} P(t) d t}
$$

donde $\mathrm{P}(\mathrm{t})$ es el valor del parámetro medido al tiempo $\mathrm{t}$ (en nuestro caso $\mathrm{pH}$ ).

El primer momento de la función $\mathrm{E}(\mathrm{t})$ mide el tiempo de residencia promedio $\left(\mathrm{t}_{\mathrm{m}}\right)$ :

$$
t_{m}=\frac{\int_{0}^{\infty} t E(t) d t}{\int_{0}^{\infty} E(t) d t}
$$

mientras que el segundo momento corresponde al valor de la varianza $\left(\sigma^{2}\right)$ :

$$
\sigma^{2}=\int_{0}^{\infty}\left(t-t_{m}\right)^{2} E(t) d t
$$

\section{d2) Balances de masa - Reactor en estado estacionario \\ d2.1) Reactor como un todo}

Asumiendo que: a) la densidad de la solución permanece constante durante todo el proceso; b) el reactor opera en forma isotérmica; c) la reacción es de primer orden e irreversible; d) el reactor se comporta como un reactor tanque agitado continuo (CSTR); e) el caudal volumétrico es constante; se pueden plantear las siguientes ecuaciones:

$$
\begin{aligned}
& F_{A M}^{e}=F_{A M}^{S}+r_{A M} \cdot V \\
& F_{A M}^{S}=F_{A M}^{e}-X \cdot F_{A M}^{e} \\
& F_{A M}^{e, s}=C_{A M}^{e, s} \cdot Q \\
& \tau=\frac{V}{Q} \\
& r_{A M}=k_{1} C_{A M} \\
& D a=k_{1} C_{A M}^{e(n-1)} \cdot \tau \\
& X=\frac{D a}{D a+1}
\end{aligned}
$$

Donde:

$$
\mathrm{F}=\text { flujo másico (moles/tiempo) }
$$


e / s = entrada / salida

$\mathrm{AM}=$ anaranjado de metilo

$r=$ velocidad de reacción (moles/volumen/tiempo)

$\mathrm{V}=$ volumen del reactor $(\mathrm{L})$

$\mathrm{X}=$ conversión (adimensional)

$Q=$ caudal (volumen/tiempo)

$\tau=$ tiempo espacial

k1 = constante cinética de primer orden (1/tiempo)

$\mathrm{C}=$ concentración (moles/L)

$\mathrm{n}=$ orden de la reacción (adimensional)

$\mathrm{Da}=$ número adimensional de Damkohler

\section{d2.2) Reactor considerando las unidades por separado}

Debido a que cada unidad del reactor posee potencia y volumen diferente, se plantea la siguiente ecuación que expresa la relación funcional entre la constante cinética de primer orden y la potencia ultrasónica por unidad de volumen para la unidad i:

$$
k_{1 i}=K_{i} \frac{P_{i}}{V_{i}}
$$

Donde:

$\mathrm{P}$ = potencia ultrasónica transmitida a la solución

$\mathrm{K}=$ constante de velocidad intrínseca (22)

Utilizando las ecuaciones anteriores, la concentración en la unidad i será:

$C_{A M, i}=\frac{C_{A M ; i-1}}{\left(1+\tau_{i} K_{i} \frac{P_{i}}{Q}\right)}$

y haciendo:

$\tau_{i} K_{i} \frac{P_{i}}{Q}=\propto_{i}$

$C_{A M, i}=\frac{C_{A M, i-1}}{\left(1+\propto_{i}\right)}$

Con lo cual la concentración a la salida del reactor vendrá dada por:

$$
C_{A M, 4}=\frac{C_{A M}^{e}}{\left(1+\alpha_{1}\right)\left(1+\alpha_{2}\right)\left(1+\alpha_{3}\right)\left(1+\alpha_{4}\right)}
$$

Los parámetros $\alpha_{i}$ se corresponden con los números de Damkohler de cada unidad $\left(D_{a}\right)$, mediante los cuales puede calcularse el correspondiente al reactor total (Da) mediante la ecuación (16).

$$
D a=\left[\prod_{i=1}^{4}\left(D a_{i}+1\right)\right]-1
$$




\section{d3) Balance de masa - Reactor en estado transiente}

Operando durante el transiente, la ecuación del balance de masa para la unidad i (teniendo en cuenta una cinética de primer orden) será:

$$
Q \cdot C_{A M, i-1}=Q \cdot C_{A M, i}+K_{i} \cdot P_{i} \cdot C_{A M, i}+V_{i} \cdot \frac{d C_{A M, i}}{d t}
$$

Se tienen entonces cuatro ecuaciones donde la condición inicial $(\mathrm{t}=0)$ para cada unidad es $C_{A M, i}=C_{A M}^{e}$, debido a que al comienzo de la operación todas las unidades se encuentran llenas con la solución de entrada a la unidad 1.

\section{d4) Balance de masa - Reactor en estado transiente y estable}

Con el fin de modelar el funcionamiento del reactor teniendo en cuenta ambos estados, la ecuación (17) se transforma en:

$$
Q \cdot C_{A M, i-1}=Q \cdot C_{A M, i}+K_{i} \cdot P_{i} \cdot C_{A M, i}+V_{i} \cdot \frac{d C_{A M, i}}{d t} \cdot H(t-\sigma)
$$

donde $H(t-\sigma)$ es la función escalón de Heaviside, $\sigma$ es el valor del tiempo en que el reactor pasa del estado transiente al estado estable y $\mathrm{H}$ toma el valor 1 para tiempos menores a $\sigma$ y cero si es igual o mayor a dicho valor.

\section{RESULTADOS Y DISCUSION}

a) Distribución del tiempo de residencia

Dado que el fluido utilizado fue agua destilada (cuyos valores iniciales de $\mathrm{pH}$ promedios se indican en la Tabla 1) y el trazador fue $\mathrm{HCl}$, con el fin de calcular los momentos de la función del tiempo de residencia, a los valores iniciales de pH se les restó cada uno de los valores medidos a la salida de cada unidad, obteniéndose gráficos como los mostrados en la Figura 2 para el caso de la unidad 1. Estos datos fueron utilizados para obtener las áreas debajo de las curvas mediante el método de los trapecios obteniéndose a través de las ecuaciones 1-3 los valores de la función de DTR y sus dos primeros momentos (Tabla 2)

Tabla 1. Valores iniciales de $\mathrm{pH}$ en cada unidad

\begin{tabular}{|c|c|c|}
\hline Unidad & pH promedio & Desvío estándar \\
\hline 1 & 7,65 & 0,23 \\
\hline 2 & 7,63 & 0,05 \\
\hline 3 & 7,70 & 0,07 \\
\hline 4 & 7,83 & 0,15 \\
\hline
\end{tabular}




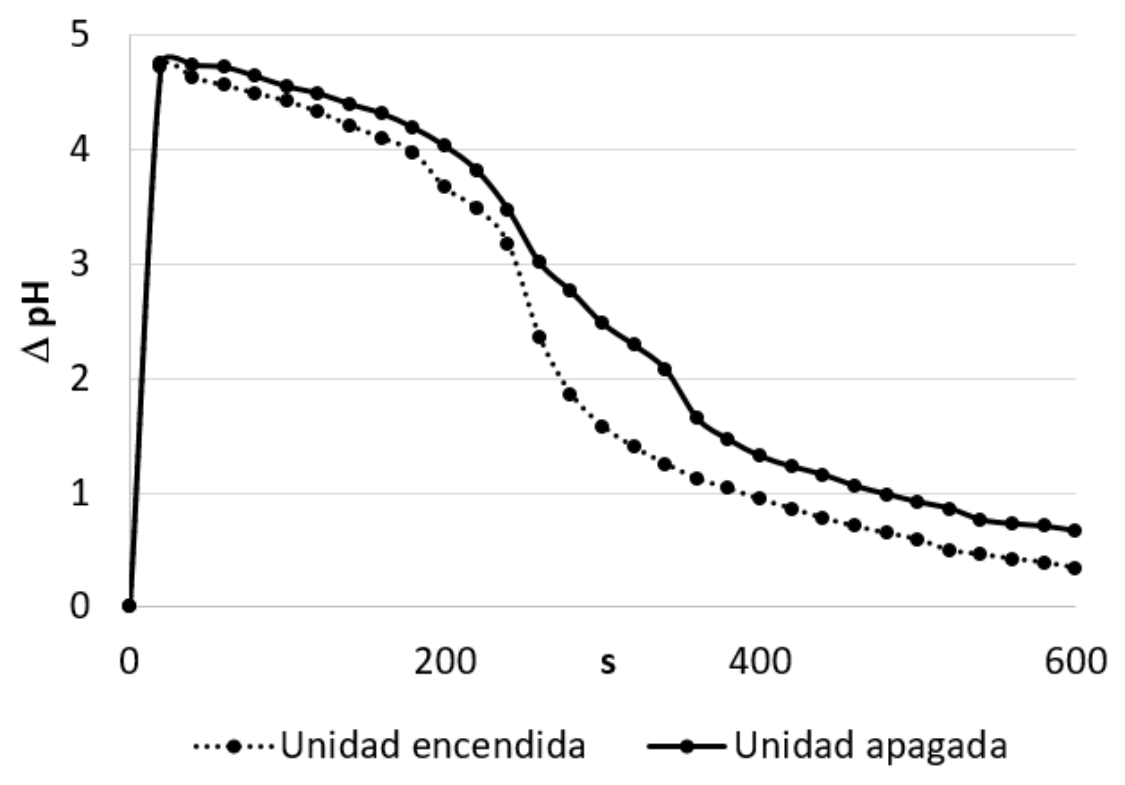

Figura 2. Diferencias de pH en función del tiempo para la unidad 1

Tabla 2. Tiempo de permanencia (o espacial) $(\tau)$, tiempo de residencia promedio $\left(t_{m}\right)$ y desvío estándar $(\sigma)$ para cada unidad.

\begin{tabular}{|c|c|c|c|c|}
\hline Unidad & $\begin{array}{c}\tau \\
(\mathbf{m i n})\end{array}$ & $\begin{array}{c}\text { Estado del sono- } \\
\text { trodo }\end{array}$ & $\begin{array}{c}\mathbf{t}_{\mathbf{m}} \\
(\mathbf{m i n})\end{array}$ & $\begin{array}{c}\boldsymbol{\sigma} \\
(\mathbf{m i n})\end{array}$ \\
\hline \multirow{2}{*}{1} & 3,18 & Encendido & 3,05 & 2,17 \\
\cline { 3 - 5 } & & Apagado & 3,38 & 2,32 \\
\hline \multirow{2}{*}{2} & 3,16 & Encendido & 3,17 & 2,25 \\
\cline { 3 - 5 } & & Apagado & 3,08 & 2,23 \\
\hline \multirow{2}{*}{3} & \multirow{2}{*}{3,17} & Encendido & 3,20 & 2,30 \\
\cline { 3 - 5 } & \multirow{2}{*}{4} & Apagado & 3,19 & 2,28 \\
\hline & 3,21 & Encendido & 3,08 & 2,22 \\
\cline { 3 - 5 } & & Apagado & 3,46 & 2,33 \\
\hline
\end{tabular}

La distribución de los tiempos de residencia para cada una de las cuatro unidades del reactor muestra que no existen diferencias significativas entre los valores de los tiempos espaciales y el tiempo de residencia prome- 
dio, tanto para el reactor encendido como apagado. Esto indica que el reactor presenta una muy buena aproximación a un reactor de mezcla completa en el cual no existen volúmenes muertos, recirculaciones o cortocircuitos que afecten el normal funcionamiento del mismo.

b) Reactor en estado estable - Reactor como un todo

Utilizando los datos de ensayos realizados con anaranjado de metilo para cuatro concentraciones iniciales diferentes (20) y mediante la hoja de cálculo Open Office 4 y las ecuaciones $4-10$, se obtuvieron los valores de los parámetros del reactor (Tabla 3), los cuales muestran dependencia de las concentraciones de entrada. Mediante el programa Curve Expert Professional 2.6 se determinó la siguiente relación entre los valores de las constantes de velocidad y las concentraciones de entrada:

$k_{1}=\frac{\left(a+b \cdot C_{A M}^{e}\right)}{\left(1+c . C_{A M}^{e}+d \cdot\left(C_{A M}^{e}\right)^{2}\right)}$

donde $\mathrm{a}=8,740749 \mathrm{E}-01(1 / \mathrm{min}), \mathrm{b}=1,231719 \mathrm{E} 01$ ( $\mu \mathrm{mol} / \mathrm{min} \cdot \mathrm{ml}), \mathrm{c}=-2,395164 \mathrm{EO} 2(\mu \mathrm{mol} / \mathrm{min} \cdot \mathrm{ml}), \mathrm{d}=$ $1,372835 \mathrm{E} 04\left((\mu \mathrm{mol} / \mathrm{min} \cdot \mathrm{ml})^{2}\right)$ y un valor del coeficiente de determinación $\left(R^{2}\right)$ de 1.0000 .

Tabla 3. Parámetros del reactor para cuatro concentraciones de entrada.

\begin{tabular}{|c|c|c|c|}
\hline $\begin{array}{c}C_{A M}^{e} \\
(\mu \mathrm{mol} / \mathrm{L})\end{array}$ & $\begin{array}{l}k 1 * 10^{3} \\
(1 / \mathrm{min})\end{array}$ & $\begin{array}{c}\text { rj } \\
(\mu \mathrm{mol} / \mathrm{min} / \mathrm{L})\end{array}$ & $x$ \\
\hline 26,554 & 278,049 & 1,627 & 0,780 \\
\hline 21,222 & 540,717 & 1,457 & 0,873 \\
\hline 15,396 & 1877,829 & 1,162 & 0,960 \\
\hline 10,837 & 60609,349 & 0,851 & 0,999 \\
\hline
\end{tabular}

Por otra parte, entre la fracción de conversión y la concentración de entrada, se ajustó la siguiente ecuación:

$$
\begin{aligned}
& \mathrm{X}=\mathrm{m}+\mathrm{n} . C_{A M}^{e} \\
& \text { con } \mathrm{m}=1,163940, \mathrm{n}=-14,114004 \text { y } \mathrm{R}^{2}=0,9828 .
\end{aligned}
$$

A partir de las ecuaciones 4, 6, 7 y 8 (que tienen en cuenta únicamente las concentraciones de ingreso a la unidad 1 y egreso de la unidad 4) y empleando el módulo XCOS de Scilab, se construyó el modelo dinámico 
del reactor (Figura 3). Utilizando el mismo se calcularon las concentraciones de salida (Tabla 4), para las cuatro concentraciones de AM.

Tabla 4. Concentraciones de entrada $\left(C_{A M}^{e}\right)$, salida $\left(C_{A M}^{S}\right)$ y desvío estándar de las concentraciones de salida $\left(\sigma_{C s}\right)$ medidas y concentraciones calculadas mediante el modelo dinámico $\left(C_{A M, \text { calc }}^{S}\right)$

\begin{tabular}{|c|c|c|c|}
\hline $\begin{array}{c}\boldsymbol{C}_{A M}^{\boldsymbol{e}} \\
\mu \mathrm{mol} / \mathrm{L}\end{array}$ & $\begin{array}{c}\boldsymbol{C}_{A M}^{\boldsymbol{S}} \\
\mu \mathrm{mol} / \mathrm{L}\end{array}$ & $\begin{array}{c}\sigma_{\mathrm{cs}} \\
\mu \mathrm{mol} / \mathrm{L}\end{array}$ & $\begin{array}{c}\boldsymbol{C}_{A M, \text { calc }}^{\boldsymbol{s}} \\
\mu \mathrm{mol} / \mathrm{L}\end{array}$ \\
\hline 26,554 & 5,853 & 1,384 & 5,853 \\
\hline 21,222 & 2,694 & $3,006 \mathrm{E}-01$ & 2,694 \\
\hline 15,396 & 0,619 & $4,944 \mathrm{E}-02$ & 0,618 \\
\hline 10,837 & 0,014 & $1,911 \mathrm{E}-02$ & 0,014 \\
\hline
\end{tabular}

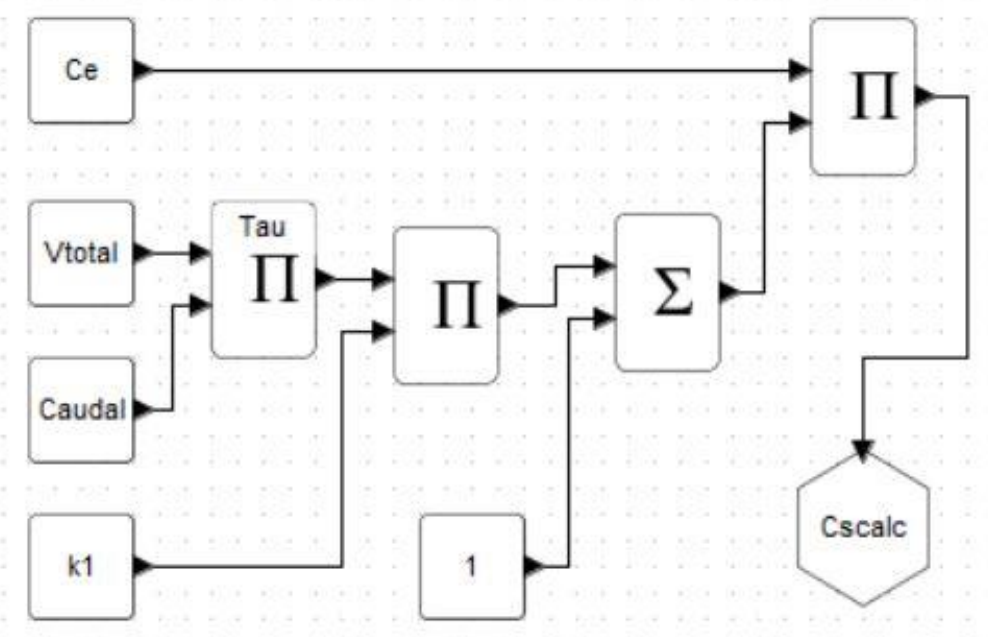

Figura 3. Modelo dinámico del reactor como un todo.

Los resultados obtenidos con el modelo (Tabla 4) concuerdan muy bien con los valores medidos. El porcentaje de conversión (Tabla 3), inversamente proporcional a la concentración de entrada, muestra que al aumentar la misma sería necesario recircular parte del caudal de salida a fin de incrementar la conversión. Para analizar el cambio en el rendimiento del reactor con dicha recirculación, se modificó el modelo dinámico como se muestra en la Figura 4, para el caso de la mayor concentración analizada ( $26.554 \mu \mathrm{mol} / \mathrm{L})$. En la misma se adiciona al caudal de entrada parte del caudal se salida, pero de manera que su suma sea igual a la utilizada 
anteriormente (la Figura 4 muestra el caso de una recirculación del 5\%). A fin de tener en cuenta que la constante de velocidad es función de la concentración de entrada, se utilizó la ecuación (19). Incrementando el porcentaje de recirculación del caudal de salida hasta un 50\%, se llega a un rendimiento del 100\% (Tabla 5).

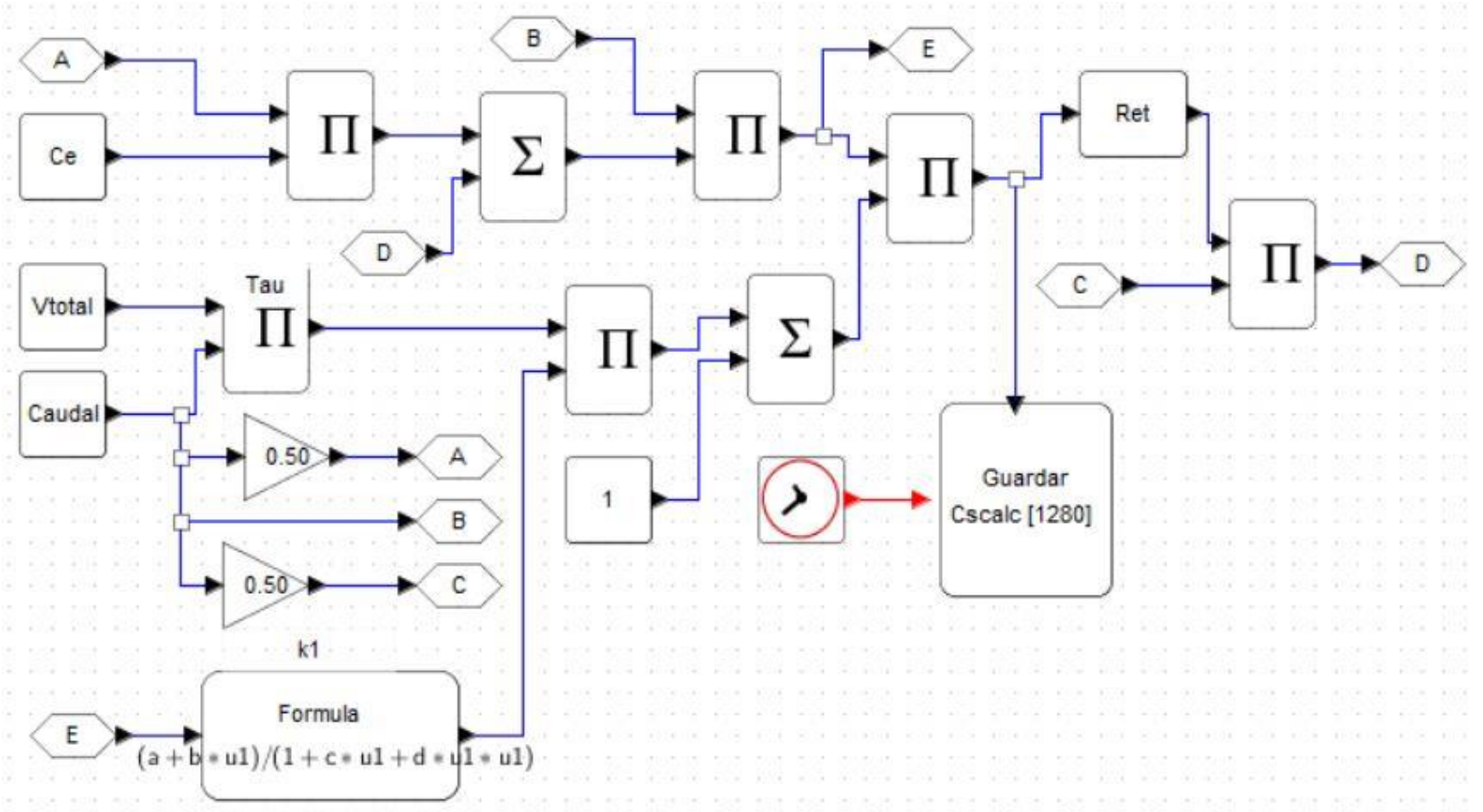

Figura 4. Modelo dinámico del reactor como un todo con recirculación de parte del caudal de salida.

Tabla 5. Variación de la conversión (X) con diferentes porcentajes de recirculación del caudal de salida (\%)

\begin{tabular}{|l|l|l|l|l|l|l|l|l|l|}
\hline$\%$ & 0 & 5 & 10 & 15 & 20 & 25 & 30 & 40 & 50 \\
\hline $\mathbf{X}$ & 0,78 & 0,81 & 0,83 & 0,86 & 0,89 & 0,91 & 0,93 & 0,97 & 0,99 \\
\hline
\end{tabular}

b) Reactor en estado estable - Reactor considerando las unidades por separado

Las relaciones determinadas entre las constantes intrínsecas y las concentraciones de ingreso a cada unidad, junto con los coeficientes de determinación se presentan en las ecuaciones (21) a (24).

$$
\begin{array}{lll}
\text { Unidad 1: } & K_{1}=3,451440 E-05 \cdot C_{e}^{-1,665426} & \mathrm{R}^{2}=0,989 \\
\text { Unidad 2: } & K_{2}=1,002187 E-04 \cdot C_{s 1}^{-1,5024681} & \mathrm{R}^{2}=0,992 \\
\text { Unidad 3: } & K_{3}=3,4824156 E-05 \cdot C_{s 2}^{-1,6448773} & \mathrm{R}^{2}=0,981 \\
\text { Unidad 4: } & K_{4}=7,4499665 E-03 \cdot C_{s 3}^{-0,61227624} & \mathrm{R}^{2}=0,996
\end{array}
$$


Usando las ecuaciones (11-14 y 21 -24) se realizó el modelo de simulación del reactor teniendo en cuenta cada unidad (Figura 5 y Tabla 6). Mediante el test de Student se realizó la comparación estadística entre los valores de las concentraciones de salida determinadas analíticamente y las calculadas mediante el modelo dinámico. Los resultados (Tabla 7) muestran que no existen diferencias estadísticamente significativas entre ambos valores, aunque el ajuste sería inferior al obtenido considerando al reactor como un todo debido a los errores en el cálculo de las constantes de reacción intrínsecas (varianza no explicada $=1-R^{2}$ ).
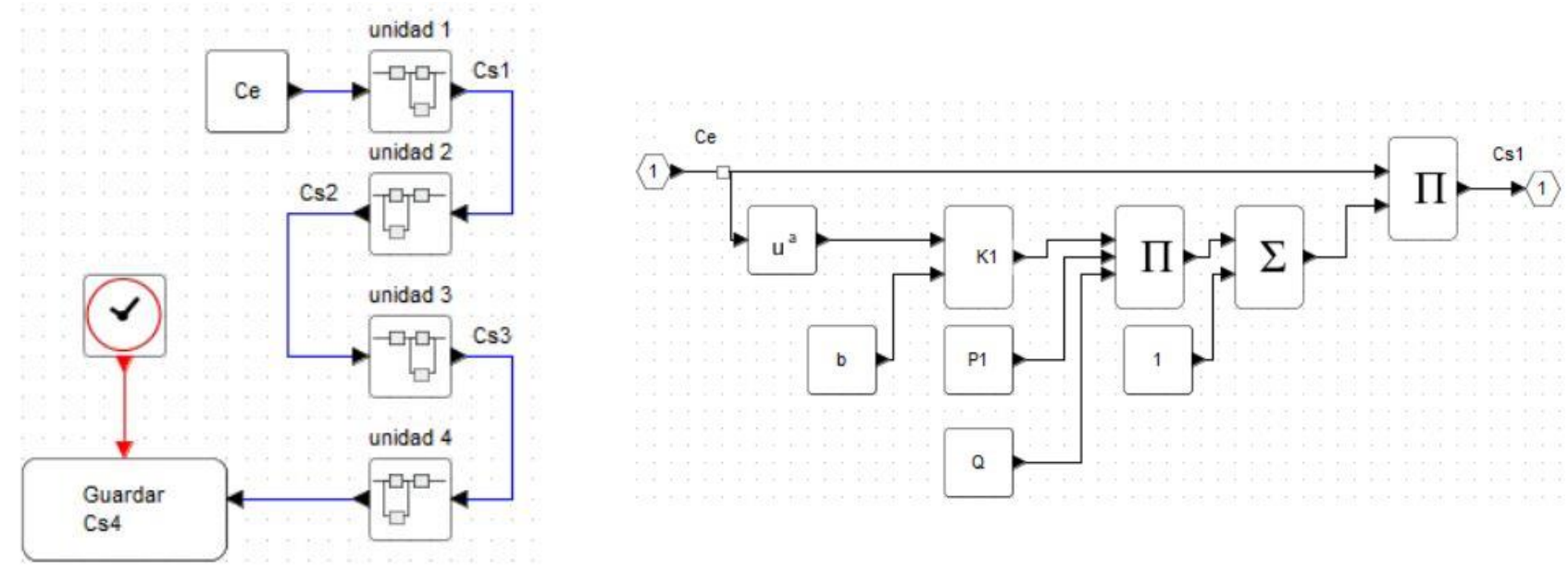

Figura 5. Simulación dinámica. Izquierda: Reactor con sus cuatro unidades. Derecha: detalle de la unidad 1

Tabla 6. Valores determinados $\left(C_{A M}^{S}\right)$ y calculados $\left(C_{A M, c a l c}^{S}\right)$ mediante el modelo dinámico teniendo en cuenta las cuatro unidades del reactor

\begin{tabular}{|c|c|c|}
\hline $\begin{array}{c}\boldsymbol{C}_{A M}^{e} \\
\mu \mathrm{mol} / \mathrm{L}\end{array}$ & $\begin{array}{c}\boldsymbol{C}_{A M}^{S} \\
\mu \mathrm{mol} / \mathrm{L}\end{array}$ & $\begin{array}{c}\boldsymbol{C}_{A M, \text { calc }}^{\boldsymbol{s}} \\
\mu \mathrm{mol} / \mathrm{L}\end{array}$ \\
\hline 26,554 & 5,853 & 5,407 \\
\hline 21,222 & 2,694 & 2,521 \\
\hline 15,396 & 0,619 & 0,459 \\
\hline 10,837 & 0,014 & 0,016 \\
\hline
\end{tabular}


Tabla 7. Test de Student de las diferencias entre los valores de salida determinados y calculados mediante el modelo dinámico

\begin{tabular}{|c|c|c|c|}
\hline $\begin{array}{c}\text { Promedio de las dife- } \\
\text { rencias }(\mu \mathrm{mol} / \mathrm{L})\end{array}$ & $\begin{array}{c}\text { Desvío estándar de } \\
\text { las diferencias } \\
(\mu \mathrm{mol} / \mathrm{L})\end{array}$ & $\mathbf{t}_{\mathbf{0}}$ calculado & $\mathbf{t}(\mathbf{9 7 . 5 , 3})$ tabulado \\
\hline 0.194 & 0.291 & 1.334 & 3.183 \\
\hline
\end{tabular}

Calculando los números de Damkohler teniendo en cuenta el reactor como un todo (mediante la ecuación 9) y considerando a cada unidad por separado (utilizando la ecuación 15) se observa (Tabla 8) que los valores en ambos casos y para todas las concentraciones iniciales empleadas, muestran valores de $\mathrm{Da}>>1$ indicando que las velocidades de reacción son muy superiores a las velocidades de dispersión.

\begin{tabular}{|c|c|c|}
\hline $\boldsymbol{C}_{\boldsymbol{A M}}^{\boldsymbol{e}}(\boldsymbol{\mu \mathrm { mol } / \mathrm { L } )}$ & Da (ecuación 9) & Da (ecuación 15) \\
\hline 26,554 & 3,537 & 3,537 \\
\hline 21,222 & 6,878 & 6,875 \\
\hline 15,396 & 23,886 & 23,886 \\
\hline 10,837 & 770,951 & 750,043 \\
\hline
\end{tabular}

Tabla 8. Valores del número de Damkohler

Utilizando el test de Student (suponiendo varianzas diferentes), se demuestra que no existen diferencias estadísticamente significativas entre su cálculo teniendo en cuenta el reactor como un todo y cada una de las unidades por separado (Tabla 9).

Tabla 9. Comparación de los números de Damkohler mediante el test de Student

\begin{tabular}{|c|c|c|c|}
\hline $\begin{array}{c}\text { Promedio de las dife- } \\
\text { rencias }\end{array}$ & $\begin{array}{c}\text { Desvío estándar de } \\
\text { las diferencias }\end{array}$ & $\mathbf{t}_{\mathbf{0}}$ calculado & $\mathbf{t}(\mathbf{9 7 , 5 ; 6 )}$ tabulado \\
\hline 5,228 & 264,935 & 0,020 & 2,447 \\
\hline
\end{tabular}


c) Reactor en estado transiente - Reactor considerando las unidades por separado

La estructura del modelo de simulación del reactor es similar a la mostrada a la izquierda en la Figura 5, pero difieren las correspondientes a las unidades, dado que se agrega el término de variación temporal de la concentración, que es necesario integrar. En la Figura 6 se presenta el diagrama correspondiente a la unidad 1. El análisis estadístico de las diferencias observadas para cada una de las unidades, utilizando el test de Student para muestras independientes con varianzas diferentes, entre las concentraciones medidas y las obtenidas mediante el modelo muestran buen ajuste al modelo para todos los casos (Tabla 10).

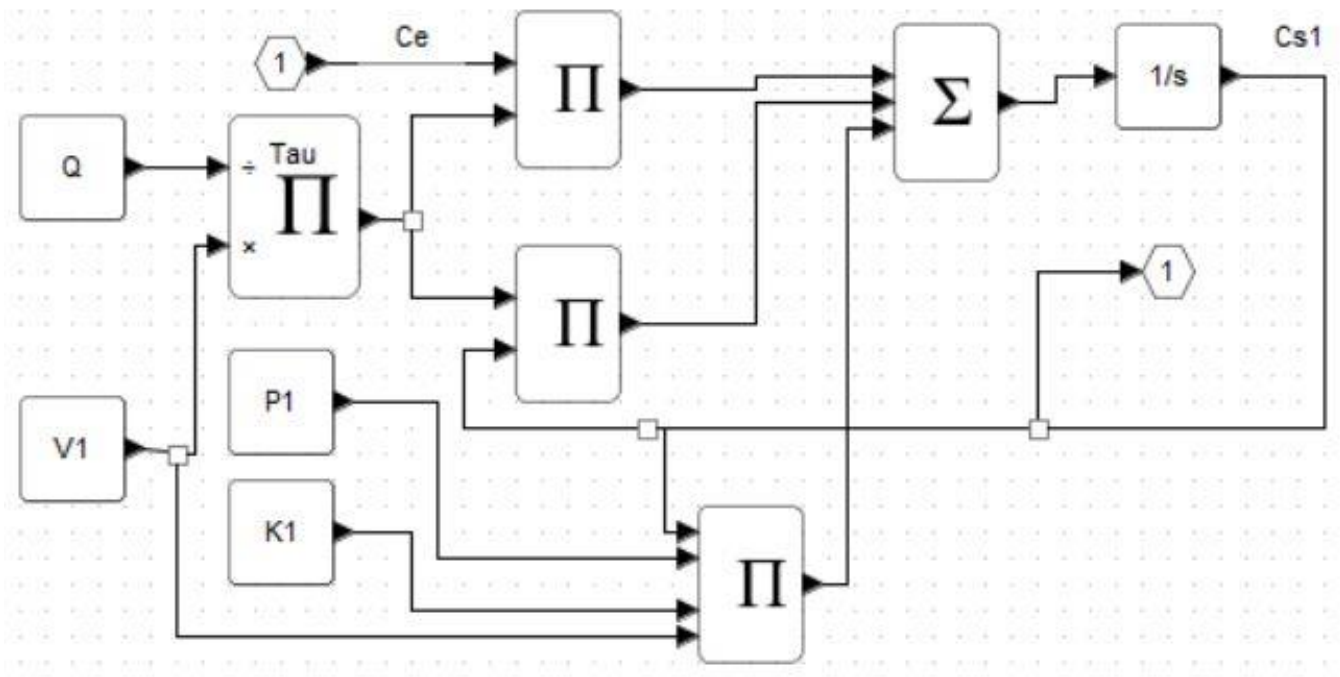

Figura 6. Modelo de simulación de la unidad 1 para el estado transiente.

Tabla 10. Test de Student entre los valores de las concentraciones de cada unidad medidas en el transiente y las calculadas mediante el modelo dinámico; dmedia= diferencia entre los valores medios $(\mu \mathrm{mol} / \mathrm{L}) ; \sigma \mathrm{d}=$ desvío estándar de las diferencias $(\mu \mathrm{mol} / \mathrm{L}) ; \mathrm{g} . \mathrm{l} .=$ grados de libertad; tcalc $=$ estadístico calculado; ttab = estadístico tabulado $(\alpha / 2$, g.l.),$\alpha=5 \%$.

\begin{tabular}{|c|c|c|c|c|c|c|}
\hline$C_{A M}^{e}(\mu \mathrm{mol} / \mathrm{L})$ & Unidad & dmedia & $\sigma d$ & g.l. & tcalc & ttab \\
\hline \multirow{4}{*}{26,553} & 1 & $5,360 \mathrm{E}-01$ & 1,033 & \multirow{4}{*}{8} & 0,519 & \multirow{4}{*}{2,447} \\
\hline & 2 & 6,406 E-01 & 2,001 & & 0,320 & \\
\hline & 3 & 1,062 & 2,948 & & 0,360 & \\
\hline & 4 & 2,345 & 5,516 & & 0,425 & \\
\hline \multirow{3}{*}{21,222} & 1 & $4,576 \mathrm{E}-01$ & 2,200 & \multirow{3}{*}{4} & 0,208 & \multirow{3}{*}{2,776} \\
\hline & 2 & $1,621 \mathrm{E}-01$ & 3,815 & & 0,042 & \\
\hline & 3 & $2,851 \mathrm{E} 01$ & 27,431 & & 1,039 & \\
\hline
\end{tabular}




\begin{tabular}{|c|c|c|c|c|c|c|}
\hline & 4 & $8,853 \mathrm{E}-01$ & 8,341 & & 0,106 & \\
\hline \multirow{4}{*}{15,396} & 1 & 2,329E-01 & 1,070 & \multirow{4}{*}{10} & 0,218 & \multirow{4}{*}{2,228} \\
\hline & 2 & $2,006 \mathrm{E}-01$ & 1,988 & & 0,101 & \\
\hline & 3 & 1,084 & 2,771 & & 0,391 & \\
\hline & 4 & $4,023 E-01$ & 3,445 & & 0,117 & \\
\hline \multirow{4}{*}{10,837} & 1 & $2,716 \mathrm{E}-02$ & 1,685 & \multirow{4}{*}{6} & 0,016 & \multirow{4}{*}{2,447} \\
\hline & 2 & 2,647 & 2,816 & & 0,094 & \\
\hline & 3 & $2,776 \mathrm{E}-01$ & 3,559 & & 0,078 & \\
\hline & 4 & $1,510 \mathrm{E}-03$ & 3,722 & & $4,0 \mathrm{E}-04$ & \\
\hline
\end{tabular}

d) Reactor en estado transiente y estable - Reactor considerando las unidades por separado

El modelo dinámico tiene en cuenta la anulación del término de acumulación al pasar del estado transiente al estable, lo que modifica el esquema de cada unidad de acuerdo a como se muestra en la Figura 7 . La simulación correspondiente a una concentración inicial de 15,396 $\mu \mathrm{mol} / \mathrm{L}$ de anaranjado de metilo, muestra buena relación con datos analíticos obtenidos para dicha concentración (promedios de tres medidas) para cada unidad del reactor (Figura 8).

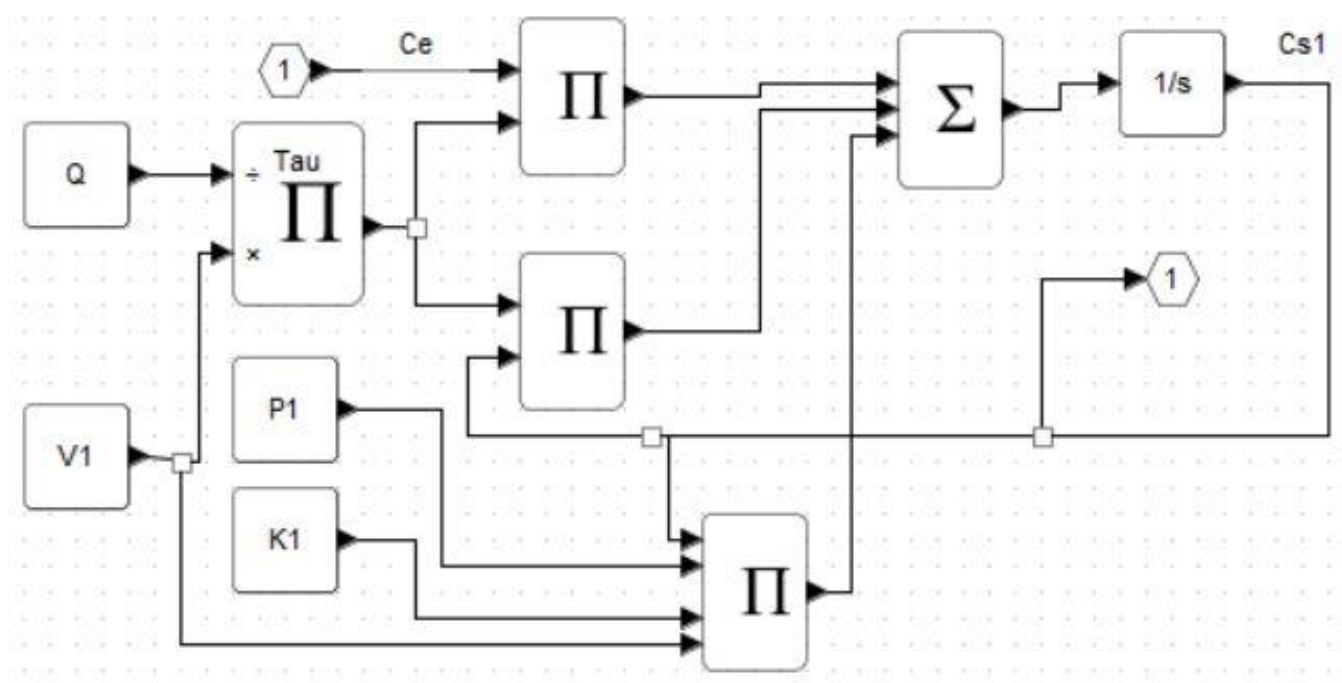

Figura 7. Modelo dinámico de la unidad 1 para los estados transiente y estable. 


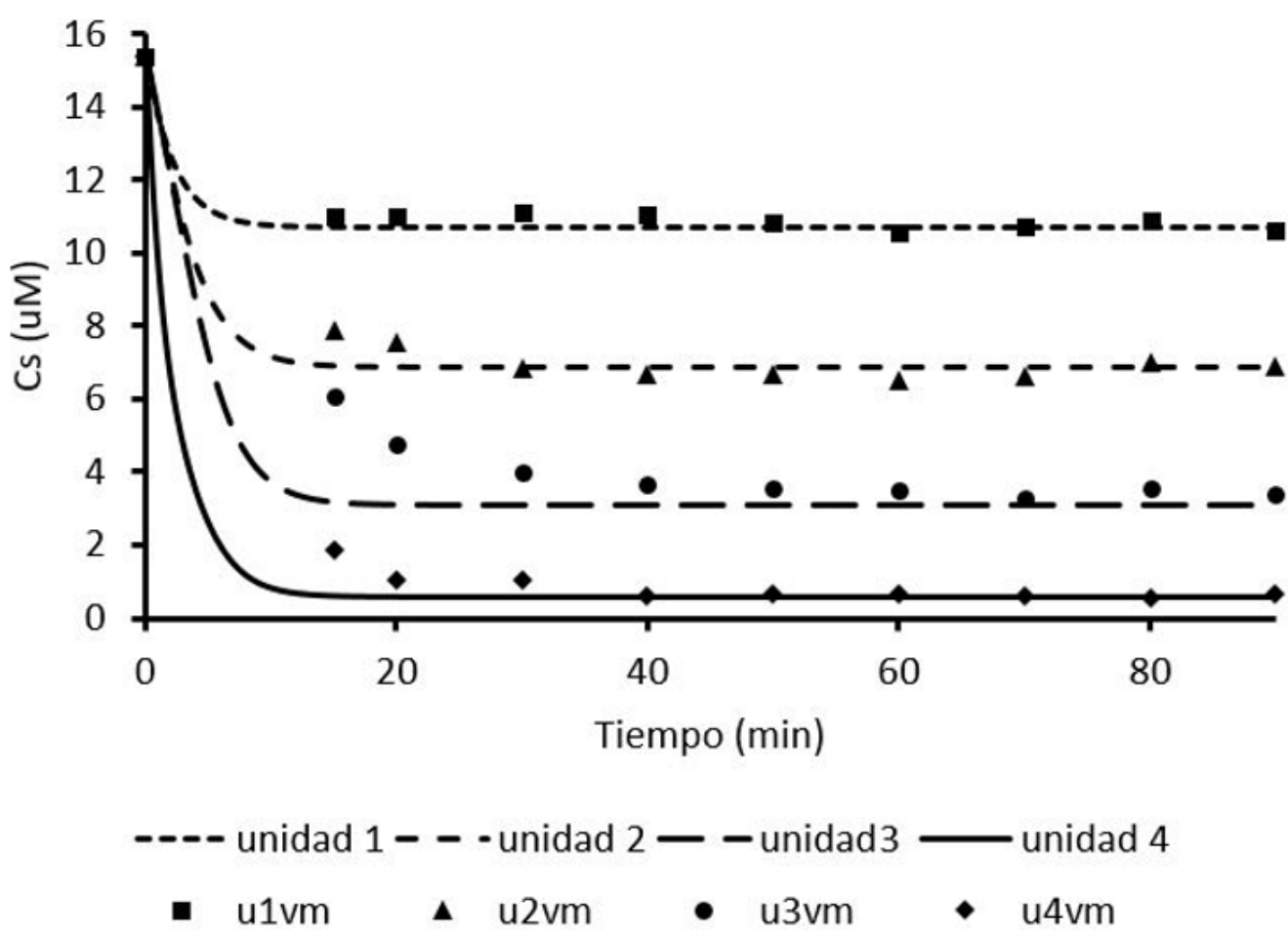

Figura 8. Resultados de la simulación para los estados transiente y estable. Líneas: valores simulados; símbolos: valores medidos (u1vm: unidad 1 - valores medidos)

El modelo dinámico simula muy bien las concentraciones del estado estable de cada una de las unidades, pero la velocidad de decaimiento simulada en el estado transiente (entre $t=0 \mathrm{~min}$ y $\mathrm{t}=30 \mathrm{~min}$ ) es superior a la obtenida a través de los datos analíticos (si bien las concentraciones no presentan diferencias estadísticamente significativas y los valores de los coeficientes de determinación $\left(R^{2}=0,973\right.$ (unidad 1), 0,974 (unidad 2), 0,896 (unidad3) y 0,989 (unidad 4) lo confirman).

Si se analiza la relación existente entre las fracciones de conversión calculadas mediante el modelo y las medidas experimentalmente para cuatro concentraciones diferentes de anaranjado de metilo, tanto para cada unidad por separado como para el reactor total (Figura 9), puede observarse que para el reactor total el rendimiento disminuye con el aumento de la concentración inicial, mientras que teniendo en cuenta las unidades por separado, independiente de la concentración inicial, el rendimiento aumenta desde la primera a la cuarta unidad; dado que las unidades son muy similares, esto también estaría mostrando una relación inversa entre rendimiento y concentración. 


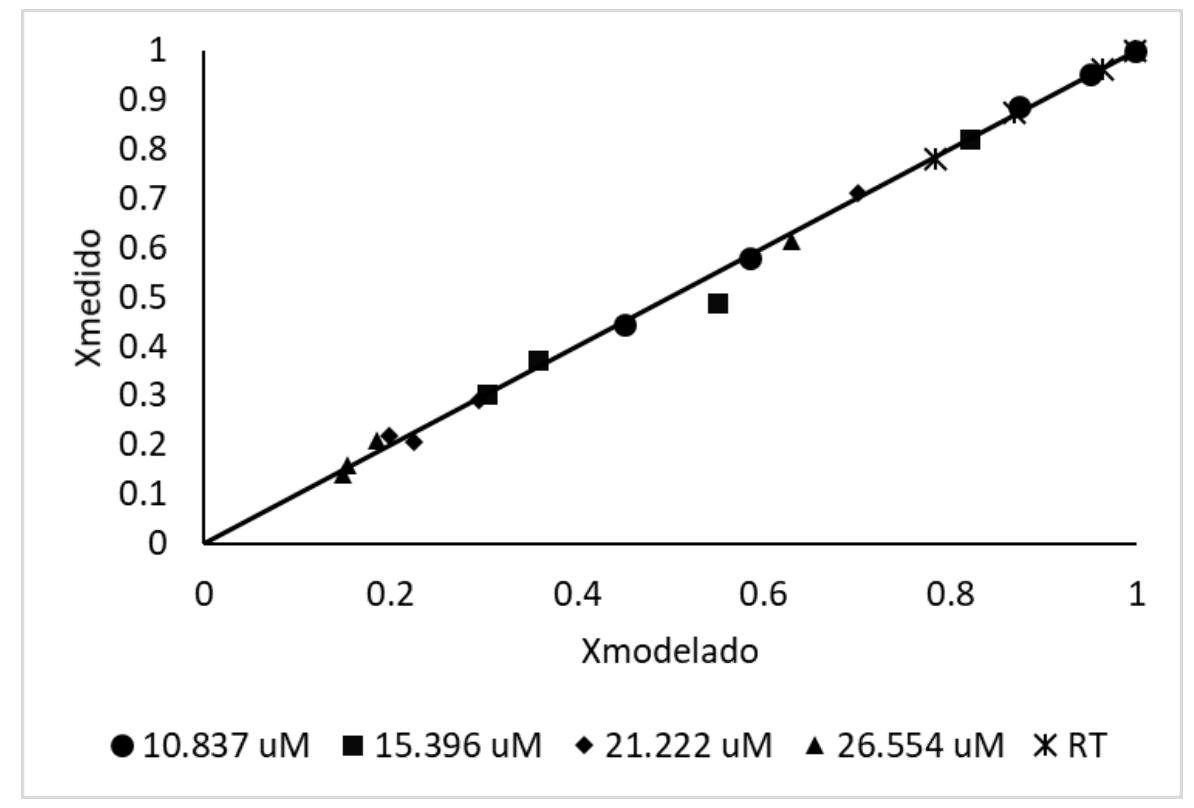

Figura 9. Comparación de las fracciones de conversión de cada unidad para cuatro concentraciones iniciales de AM y del reactor total (RT).

\section{CONCLUSIONES}

Los tiempos de residencia promedio determinados fueron similares a los tiempos espaciales lo cual indica que cada unidad se comporta como un reactor tanque continuo agitado completamente mezclado, con lo cual todo el reactor tendrá idéntico comportamiento. Las simulaciones para el estado estacionario, teniendo en cuenta el reactor como un todo o cada unidad por separado mostraron valores que no difieren estadísticamente de las determinadas analíticamente. Lo mismo sucedió para el estado transiente, aunque el ajuste fue inferior. Para el caso de la mayor concentración analizada y mediante el modelo de simulación puede inferirse que recirculando más del $20 \%$ del caudal de salida, se obtendrían fracciones de conversión superiores al 90\% (teniendo en cuenta que, sin recirculación, las mismas son del 78\%). Existe correlación entre las fracciones de conversión medidas y las modeladas, correspondiendo a una relación inversa entre las fracciones de conversión y las concentraciones iniciales.

\section{REFERENCIAS BIBLIOGRAFICAS}

1) Adewuyi, Y.G., 2005. Sonochemistry in environmental remediation. 1. Combinative and hybrid sonophotochemical oxidation processes for the treatment of pollutants in water. Environ. Sci. Technol. 39: 3409 - 3420. DOI: 10.1021/es049138y 
2) Arroyo, J. y Flores, J., 2001. Degradación ultrasónica de contaminantes orgánicos. Rev. Per. Quim. Ing. Quim. $4,2: 3-14$

3) Chowdhury, P. y Viraraghavan, T., 2009. Sonochemical degradation of chlorinated organic compounds, phenolic compounds and organic dyes - A review. Sci. Total Environ. 407: 2474 - 2492. DOI:10.1016/j.scitotenv.2008.12.031

4) Ebrahimi, R.; Tarhande, G. y Rafiei, S., 2012. The study of ultrasonic degradation of superabsorbent hydrogels. Org. Chem. Int. 2012: 1 - 5. DOI:10.1155/2012/343768

5) Gogate, P.R., 2008. Cavitational reactors for process intensification of chemical processing applications: A critical review. Chem. Eng. Process. 47: 515 - 527. DOI:10.1016/j.cep.2007.09.014

6) Ince, N.H.; Tezcanli, G.; Belen, R.K. y Apikyan, I.G., 2001. Ultrasound as a catalyzer of aqueous reaction systems: the state of the art and environmental applications. Appl. Catal. B - Environ. 29, 167-176. DOI: 10.1016/S0926-3373(00)00224-1

7) Kieffer, L. A.; de la Sierra, P. M.; Devercelli, M.; Luna, J. A., Claret, M. y Leiz, E., 2014. Degradación de Microcistina - LR mediante ultrasonido. Influencia del pH y la concentración inicial. FABICIB 18: 83-94.

8) Kieffer, L. A.; de la Sierra, P. M.; Devercelli, M.; Luna, J. A., Claret, M. y Leiz, E., 2015. Control de Floraciones Algales Mediante Ultrasonido. Ciencia, Docencia y Tecnología 26, 50: 224 - 243.

9) Hakamada, M.; Matsuzawa, T. y Mabuchi, M., 2014. Fabrication and catalytic decoloration capacity of nanodendritic metals. Mater. Trans. 55, 3: 534 - 538. DOI: 10.2320/matertrans.MBW201310

10) Kodom, T.; Amouzou, E.; Djaneye-Bo, G. y Moctar-Bawa, L., 2012. Photocatalytic discoloration of methyl orange and indigo carmine on $\mathrm{TiO}_{2}$ (P25) deposited on conducting substrates: Effect of $\mathrm{H}_{2} \mathrm{O}_{2}$ and $\mathrm{S}_{2} \mathrm{O}_{8}$. Int. J. Chem. Technol. 4, 2: 45 - 56. DOI: 10.3923/ijct.2012.45.56

11) Tang, C.W., 2013. Study of photocatalytic degradation of methyl orange on different morphologies of $\mathrm{ZnO}$ catalysts. Modern Research in Catalysis 2, 2: 19 - 24. DOI: 10.4236/mrc.2013.22003

12) Urmi, S.A.; Kurny, A.S.W. y Gulshan, F., 2015. Decolorization of methyl orange using mill scale by photoFenton reaction. Procedia Eng. 105: 844 - 851. DOI: 10.1016/j.proeng.2015.05.100

13) Wang, Y.; Gai, L.; Ma, W.; Jiang, H.; Peng, X. y Zhao, L., 2015. Ultrasound-assisted catalytic degradation of methyl orange with $\mathrm{Fe}_{3} \mathrm{O}_{4}$ /polyaniline in near neutral solution. Ind. Eng. Chem. Res. 54: 2279-2289. DOI: $10.1021 / \mathrm{ie} 504242 \mathrm{k}$

14) Youssef, N.A.; Shaban, S.A.; Ibrahim, S.A. y Mahmoud, S.A., 2016. Degradation of methyl orange using Fenton catalytic reaction. Egyptian Journal of Petroleum, 25: 317 - 321. DOI: 10.1016/j.ejpe.2015.07.017

15) Yuan, N.; Zhang, G.; Guo, S. y Wan, Z., 2016. Enhanced ultrasound-assisted degradation of methyl orange and metronidazole by rectorite-supported nanoscale zero-valent iron. Ultrason. Sonochem., 28: 62 68. DOI: 10.1016/j.ultsonch.2015.06.029 
16) Zhao, H.; Zhang, G. y Zhang, Q., 2014. $\mathrm{MnO}_{2} / \mathrm{CeO}_{2}$ for catalytic ultrasonic degradation of methyl orange. Ultrason. Sonochem., 21, 3: 991 - 996. DOI: 10.1016/j.ultsonch.2013.12.002

17) Zyoud, A.; Zu’bi, A.; Helal, M.H.S.; Park, D.; Campet. G. y Hilal, H.S., 2015. Optimizing photo-mineralization of aqueous methyl orange by nano-ZnO catalyst under simulated natural conditions. J. Environ. Health Sci. 13: 1 - 10. DOI: 10.1186/s40201-015-0204-0

18) Taylor Technologies Inc., 2016. Safety Data Sheet. Disponible en: https://www.taylortechnologies.com/en/Image/GetDocument/20.

19) Sigma - Aldrich, 2004. Material Safety Data Sheet. Disponible en: http://www.glue.umd.edu/ choi/MSDS/Sigma-Aldrich/METHYL\%20ORANGE.pdf.

20) Kieffer, L.A.; de la Sierra, P.M.; Claret, M.; Martín, C. y Leiz, S., 2019. Decoloración sonoquímica sinérgica del anaranjado de metilo utilizando triclorometano en dos reactores ultrasónicos. Acepatado para publicar en el número 1(36) de febrero de 2020.

21) Cheng, Z.; Quan, X.; Xiong, Y.; Yang, L. y Huang, Y., 2012. Synergistic degradation of methyl orange in a ultrasound intensified photocatalytic reactor. Ultrason. Sonochem., 19: 1027-1032. DOI: 10.1016/j.ultsonch.2012.02.008

22) Gondrexon, N.; Renaudin, V.; Petrier, C.; Boldo, P.; Bernis, A. y Gonthier, Y., 1999. Degradation of pentachlorophenol aqueous solution using a continuous flow ultrasonic reactor Ultrason. Sonochem., 5: 125 $-131$. 